Preprint (5.29.18) version of: Nees, M.A. Drivers' perceptions of functionality implied by terms used to describe automation in vehicles. Presented at the annual meeting of the Hum Factors and Ergonomics Society, 1-5 October 2018, Philadelphia, PA.

\title{
Drivers' Perceptions of Functionality Implied by Terms Used to Describe Automation in Vehicles
}

\author{
Michael A. Nees \\ Lafayette College, Easton, PA, neesm@lafayette.edu
}

\begin{abstract}
The expectations induced by the labels used to describe vehicle automation are important to understand, because research has shown that expectations can affect trust in automation even before a person uses the system for the first time. An online sample of drivers rated the perceived division of driving responsibilities implied by common terms used to describe automation. Ratings of 13 terms were made on a scale from 1 ("human driver is entirely responsible") to 7 ("vehicle is entirely responsible") for three driving tasks (steering, accelerating/braking, and monitoring). In several instances, the functionality implied by automation terms did not match the technical definitions of the terms and/or the actual capabilities of the automated vehicle functions currently described by the terms. These exploratory findings may spur and guide future research on this under-examined topic.
\end{abstract}

\section{INTRODUCTION}

Automated functions-such as adaptive cruise control and emergency braking — are already available in vehicles, and the trend toward automation of driving functions is expected to continue, possibly culminating in completely autonomous, self-driving vehicles. Vehicle manufacturers, government agencies, and automotive professional organizations have attempted to develop a vocabulary to describe automated functions in vehicles, including automated systems for which the vehicle and driver share responsibility for driving tasks.

The Society of Automotive Engineers (SAE) established a set of definitions for terms that described functional capabilities for five levels of automation (SAE International, 2016). Level 1 (L1), "driver assistance," describes a system in which the automation controls either steering or accelerating/braking, though not both concurrently. Level 2 (L2), "partial automation," describes a system in which both steering and accelerating/braking are automated simultaneously. The driver always is responsible for monitoring the driving scenario in L1 and L2 systems. Level 3 (L3), "conditional automated driving," describes a system with the features of an L2 system, except the driver is not required to monitor the driving scenario. The driver is still expected to intervene and serve as a fallback upon request. Level 4 (L4), "high automation," describes a system with the characteristics of an L3 system, except the driver is not expected to serve as a fallback. L1-L4 may describe systems that are limited to use under specific conditions-limitations could include geography (e.g., "geo-fencing"), road types (e.g., highwayonly), or weather conditions. Level 5 (L5), "full automation," describes an entirely automated system that can drive in any of the same circumstances as a human driver. The SAE standard provided a descriptive set of operational definitions along a continuum of automated functions.

Still, debates and confusion about the terms that have been used to describe automation in vehicles have emerged in the popular discourse (e.g., Lambert, 2017). Tesla, for example, has drawn criticism for naming its automated functions "Autopilot." Critics (e.g., Pak, 2016) contended that the name implies greater functionality than the capabilities of the Tesla system, which requires drivers to keep their hands on the wheel and actively monitor the driving scenario when Autopilot mode is engaged. A consumer group lobbied for regulation of the use of the terms "self-driving," "automated," and "Autopilot" over concerns that the terms give "...the dangerous - and sometimes fatal - impression that a car is more capable of driving itself than is actually the case" (Simpson, 2016, pp. 1). Similarly, amidst concern about misunderstandings of the term "Autopilot," German authorities sent a letter to Tesla owners notifying them that the system requires "the constant and full attention of the user in regard to the current traffic situation" (Schmitt, 2016).

Semantic confusions can have substantive consequences if the words used to describe automated functions give drivers an inaccurate impression of the capabilities of the vehicles. Research has suggested that product names can invoke strong associations. In studies conducted by Wänke, Herrmann, \& Schaffner (2007), people inferred that a product possessed the attributes associated with its name. The influence of these associations survived both explicit instructions that refuted the associations as well as testimonials from other consumers that contradicted the associations. This research suggested that impressions created by product labels can invoke lasting expectations about the product that are difficult to alter.

The labels ascribed to automated functions in vehicles might influence drivers' expectations and understanding of a system's capabilities-and thereby influence trust-before drivers even use the system. Trust is an important predictor of the extent to which an operator will rely on automation to accomplish its intended functions in the system. Overtrust can result in complacency-failure to monitor the system as its designers intended (Parasuraman \& Manzey, 2010)—and overreliance-depending on automation to a greater extent than is warranted by its capabilities (Parasuraman \& Riley, 1997). Hoff and Bashir's (2015) model of trust identified expectations and reputational qualities of the system as factors that influence trust before automated systems are used for the first time. Thus, trust in automation may be influenced by expectations acquired before an operator interacts with an automated system for the first time.

Research also has suggested that acute safety concerns can result from unrealistic expectations about the capabilities of automation during early use of the system. Körber, Baseler, and Bengler (2018) showed that an initial (before first use) introduction that was intended to promote trust in an automated driving system resulted in several potentially problematic consequences for participants' driving behaviors 
as they began interacting with the system, including more time with eyes away from the road engaging in a secondary nondriving task, longer takeover times in a critical situation that required intervention, and shorter time-to-collision in a critical situation.

Though empirical research on the topic is scant, Abraham, Seppelt, Mehler, and Reimer, (2017) recently examined how the brand names ascribed to automated functions in vehicles impacted expectations about the capabilities of the system. Their findings showed that, with the exception of the term "active cruise control," most participants could not match the brand names of automated functions to their current level of capability on a descriptive levels-of-automation scale.

The current research sought to further understand perceptions evoked by the terms used to describe vehicle automation. Participants were presented with 13 different terms that have been used to describe automation in vehicles. For each term, participants rated the extent to which they perceived the human driver or the automated system is responsible for each of three driving tasks (steering, accelerating/braking, and monitoring the driving scenario).

\section{Participants}

\section{METHOD}

Participants $(N=301)$ were recruited online via Amazon Mechanical Turk (AMT). AMT workers were required to be located in the United States, to have a task approval rating of greater than 95\%, and to have at least 1000 previously approved tasks. The study description also indicated that the research was only open to licensed drivers. Participants were paid $\$ 1.00$ for their time. Sixty-four participants failed attention check questions (described below), so the final sample analyzed was $N=237$ (97 females, 139 males, and 1 person who chose "other/prefer not to respond"). Self-reported racial/ethnic backgrounds were white $(n=189)$, Asian $(n=$ $26)$, black or African American $(n=11)$, Hispanic or Latino ( $n$ $=18)$, American Indian or Alaska Native $(n=2)$, and Native Hawaiian or Pacific Islander $(n=3)$. Participants were allowed to select more than one category, so the sum of these numbers is greater than the sample size. Age data were inadvertently omitted due to a computer error, but participants reported $M=17.21$ years of driving experience $(S D=10.37$, $m d n=15.00$, minimum $=1.00$, maximum $=50.00)$, which is consistent with a sample with a mean age in the mid-thirties (see Nees, 2016). Participants reported driving $M=7.66$ hours per week $(S D=6.15, m d n=5.00$, minimum $=0.00$, maximum $=40.00)$. Participants reported the scenario that best described where they drive most often as urban/city driving $(n=131)$, rural/small town driving $(n=78)$, distance/interstate/freeway driving $(n=27)$, and other $(n=1)$. On a scale from 1 ("not familiar at all") to 7 ("extremely familiar"), participants rated their familiarity with the current state of technology related to vehicle automation and self-driving cars as $M=3.70(S D=$ $1.37, m d n=4.00)$. Participants reported having read $M=3.70$ articles about self-driving cars in print or online $(S D=5.56$, $m d n=2.00$, minimum $=0.00$, maximum $=50.00)$. Four participants reported owning or using a vehicle that had automated features, including one instance each of Autopilot, autobrake, driver assist, and ACC.

\section{Survey Questions and Procedure}

The survey examined 13 different terms that have been used to described automation in vehicles. The first six terms ("no automation," "driver assistance," "partial automation," "conditional automation," "high automation," and "full automation") were the names of the levels of vehicle automation in the SAE standard (SAE International, 2016). "Self-driving," "semi-autonomous," and "autonomous" were included, because they are pervasive in the general discourse on vehicle automation. Finally, four terms were included as examples of proprietary terms used by vehicle manufacturers to describe extant or prototype systems ["Autopilot," (Tesla); "copilot," (NVIDIA, BMW); "drive pilot," (formerly used by Mercedes); and "pilot assist" (Volvo)].

Participants rated the extent to which each term suggested that the driver or the vehicle was responsible for each of three dynamic driving tasks (DDTs, see SAE International, 2016): (1) steering; (2) accelerating and braking; and (3) monitoring the driving scenario. Each DDT was rated separately. Figure 1 shows an example of the phrasing and formatting of a question, the rating scale, and the anchors. For terms that worked best as adjectives (e.g. "semi-autonomous"), the questions used minor variations in phrasing to accommodate the term (e.g., "Consider a vehicle with a semi-autonomous mode).

Consider a vehicle with no automation. Please rate the extent to which the human driver or the vehicle is responsible for accomplishing the driving task of steering in the vehicle with no automation.

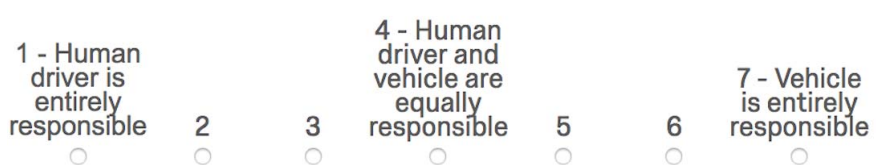

Figure 1. An example question.

Upon accepting the recruitment post on AMT, participants clicked on a link to a survey hosted on Qualtrics. Following informed consent, participants were shown instructions along with an example question. The instructions defined the three DDTs (steering, accelerating and braking, and monitoring). Participants were asked to respond based on their own impressions of the meaning of each automation term, even if they did not know the technical definition of the term. The order of presentation of the 13 automation terms was randomized for each participant. For a given term, participants saw the three DDT questions in the same order (steering, accelerating/braking, and monitoring). Two questions were included as attention checks. In one block of three DDT questions, a fourth question contained an embedded parenthetical that instructed participants, "this is a research check to be sure you are reading the questions, please select ' 5 ' as your response below." Participants who did not select " 5 " were deemed to have failed the check. In the demographic battery, participants responded to the statement, 


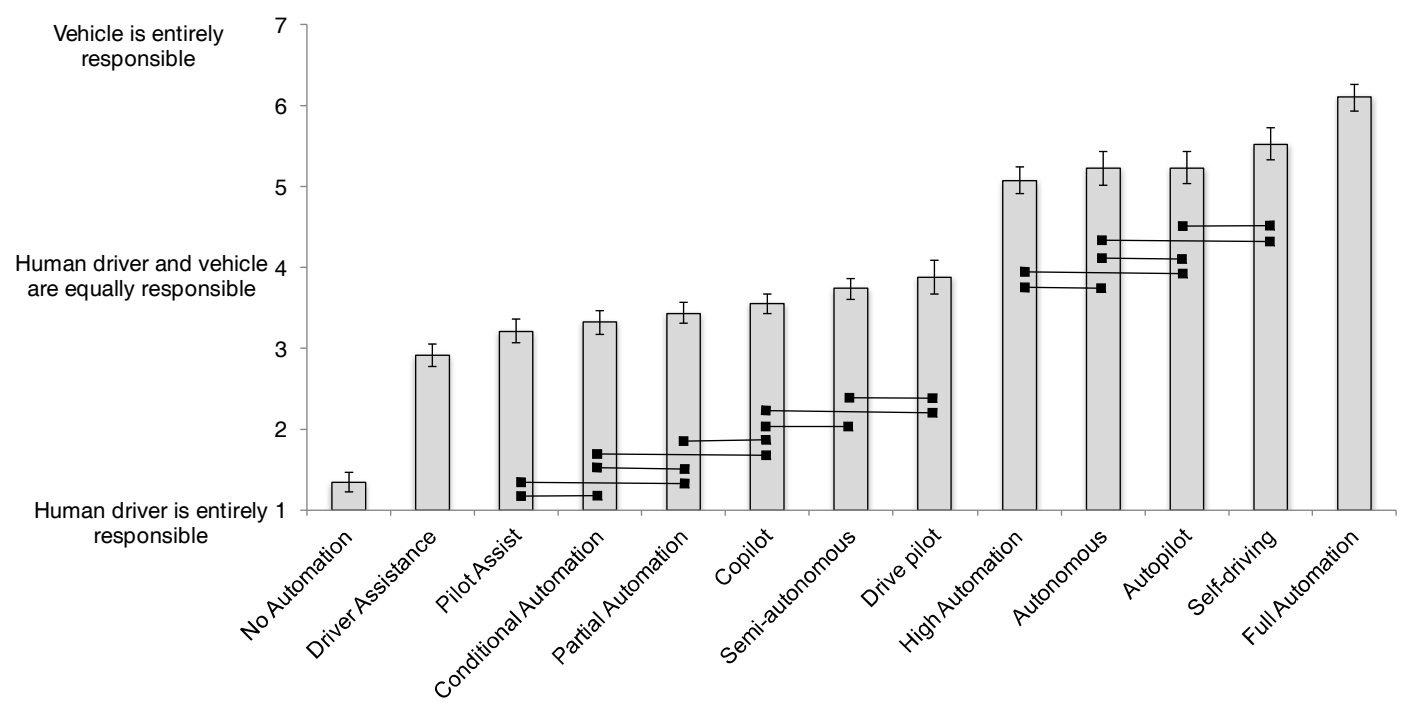

Figure 2. Post hoc comparisons for the main effect of automation term. Bar heights show mean ratings. Differences between means are significant (following Bonferroni adjustments) except for bars connected by lines. Error bars represent $95 \%$ confidence intervals.

"I followed the instructions for this study and answered all questions to the best of my ability" on a scale from 1 ("strongly disagree") to 7 ("strongly agree"). Participants who selected a response lower than "6" were deemed to have failed the check. After ratings were completed, participants answered demographic questions and were debriefed.

\section{RESULTS}

Five participants were excluded from the analyses for finishing the experiment in less than $125 \mathrm{~s}$ (range $=87$ to 124 $\mathrm{s}$ ), which suggested that they did not read each question. A $9 \mathrm{~s}$ gap separated these 5 participants from the rest of the distribution. Participants also were excluded for failing the first attention check $(n=41)$, failing the second attention check $(n=8)$, self-reporting they had no driver's license $(n=$ $5)$, or meeting more than one of these criteria $(n=5)$.

A 3 (DDT) X 13 (automation term) repeated measures analysis of variance examined ratings of responsibility. Sphericity was violated for the effects of DDT (Mauchly's $W$ $=0.67, p<.001$ ), automation term (Mauchly's $W=0.06, p$ $<.001$ ), and the interaction (Mauchly's $W<0.01, p<.001$ ), so Greenhouse-Geisser corrections were used. The ANOVA showed significant main effects of DDT, $F(1.50,353.92)=$ 51.18, $p<.001, \eta_{\mathrm{p}}^{2}=.18$, and automation term, $F(7.40$, $1747.29)=328.11, p<.001, \eta_{p}^{2}=.58$. The interaction also was significant, $F(16.24,3832.02)=10.01, p<.001, \eta_{p}^{2}=.04$.

The design afforded a large number of post hoc comparisons, so Bonferroni adjustments were used to control the Type I error rate for all follow-up tests. Post hoc comparisons to examine the significant effect of DDT showed that ratings for monitoring $(M=3.83, S E=.06)$ were significantly lower than ratings for steering $(M=4.13, S E=$ $.04)$ and accelerating/braking $(M=4.17, S E=.04), p s<.001$. Figure 2 depicts the results of post hoc comparisons for the main effect of automation term collapsed across DDTs. In all figures, error bars represent $95 \%$ confidence intervals (CIs) to allow for comparison with the Likert scale anchors (on the Yaxis). Y-axis values within the range of the error bars for a mean are plausible values of the true mean; values outside of the error bars are implausible.

Both main effects were qualified by the significant interaction. A simple effects analysis examined differences in ratings for automation terms holding DDTs constant (see Figure 3). For all DDTs, participants rated "no automation" and "full automation" as significantly different from all other terms, and these terms represented opposite ends of a continuum of perceived level of automation. Beginning with terms that were perceived to entail lower levels of automation, "driver assistance" was rated to entail more responsibility for the human than all other terms except "no automation" for all DDTs. "Pilot assist" and "conditional automation" were rated as similar for all three DDTs, and "pilot assist" and "partial automation" were rated as similar with respect to monitoring. "Partial automation" and "copilot" were rated similarly for steering and braking/accelerating, but participants perceived that "copilot" entailed less monitoring responsibility for the human than "partial automation." A "semi-autonomous" vehicle and a vehicle with "drive pilot" respectively were rated as different from all other terms regarding steering responsibilities, and these terms were different from all other terms, but not each other, with respect to accelerating/braking. A "semi-autonomous vehicle" was perceived to be the same as a vehicle with "drive pilot" and a vehicle with "copilot" with respect to monitoring responsibilities,

The terms "high automation," "autonomous," "Autopilot," and "self-driving" show a marked shift toward less perceived responsibility for the human driver for all three DDTs. The terms "high automation" and "autonomous" were rated as similar for all DDTs. "Autopilot" was rated as entailing less responsibility for the human for steering than "high automation," and it was not different from "autonomous" or "self-driving." "High automation," "autonomous," and "Autopilot" were rated similarly for both accelerating/braking and monitoring. "Self-driving" was different from all other terms and had the lowest perceived responsibility for the human driver for accelerating/braking 
except for "full automation," and "self-driving" and "autonomous" were rated similarly with respect to monitoring.

Figure 3 also depicts the alternate simple effects analysis that examined differences in the ratings of DDTs for each automation term. In general, as the perceived degree of automation increased, participants began to rate the human as relatively more responsible for monitoring as compared to steering or accelerating/braking. For both "driver assistance" and "Pilot Assist," the vehicle was rated as significantly more responsible for accelerating/braking than steering or monitoring, which could indicate that variations on the "assist" term are associated with longitudinal control of the vehicle.

\section{DISCUSSION}

This research gave an initial idea of the functionality implied to drivers by several terms that have been used in the general discourse surrounding vehicle automation. A "semiautonomous" vehicle was perceived to distribute responsibility for DDTs approximately equally between the human driver and the vehicle, although the human driver was perceived to bear relatively greater responsibility for monitoring the driving scenario than steering or accelerating/braking. Even though "autonomous" and "self-driving" both suggested relatively high levels of responsibility for the vehicle as compared to the human driver, participants still perceived that these terms imply a lower level of automation than "full automation." The term "autonomous" was equated with the term "high automation" (L4) across all driving tasks, whereas "selfdriving" was perceived to involve less responsibility for the human than "high automation" for all three DDTs. As such, the term "self-driving" appears to imply functionality somewhere between L4 ("high automation") and L5 ("full automation"). This lends some support to arguments (e.g., Lambert, 2017) that the L2 systems available in current vehicles should not be called "self-driving."

The current study partially validated that the names used in the SAE standard are perceived to indicate increasingly higher levels of automation, although there was one important exception. The terms for L2 and L3, "partial automation" and "conditional automation," were correctly situated between L1 and L4, but ratings did not match the technical definitions for L2 and L3. Specifically, for steering and accelerating/braking, participants rated the term "partial automation" (L2) as entailing a higher level of automation than "conditional automation" (L3), which is the reverse of their order according to the technical definitions. For monitoring, "partial automation" and "conditional automation" were rated similarly.

Another potentially useful set of findings emerged with respect to the proprietary terms examined in the study. At the time of this writing, the highest level of automation available in consumer vehicles is L2 (Cole, 2017). This includes Tesla's "Autopilot", Volvo's "Pilot Assist", BMW's "Personal Copilot," and Mercedes-Benz automated functions formerly called "Drive Pilot". The technical name for L2 automation is "partial automation." Of the proprietary names (or variations thereof) examined here, "Pilot Assist" and "Copilot" were perceived to be similar to "conditional automation" (L3) and "partial automation," respectively, with minor exceptions (e.g., with respect to monitoring). Since participants' ratings partially confounded "conditional" with "partial" automation, results suggested that the terms "Pilot Assist" and "Copilot" were rated similarly to the SAE terms indicating L2 and L3 automation. "Drive Pilot" generally was perceived to indicate a higher degree of automation than "Pilot assist" or "Copilot." For accelerating/braking, "Drive Pilot" was perceived as equivalent to a "semi-autonomous" vehicle. For steering, "Drive Pilot" was perceived to indicate a significantly higher degree of automation than "semi-autonomous." "Autopilot" was perceived to be equivalent to an "autonomous" and "selfdriving" vehicle for the steering DDT. "Autopilot" was perceived to be equivalent to "high automation" and "autonomous" for the DDTs of accelerating/braking and monitoring. Thus, "Autopilot" was perceived to entail the same functionality as terms such as "high automation"- - a term whose technical definition specifies greater capabilities than the current Tesla "Autopilot" functionality.

A critical step for future research is to determine the extent to which misperceptions developed before a person interacts with automated systems in vehicles will or will not result in later unsafe behavior while using automated functions in vehicles. Available evidence, though limited, has shown that the perceptions invoked by names ascribed to products can produce expectations that are resistant to new information (Wänke et al., 2007), and overly optimistic introductions to automated driving features have resulted in potentially dangerous driving behaviors (Korber et al., 2018). Some reports have suggested that current point-of-sale practices may be inadequate for training users of emerging automated systems in vehicles (see, e.g., Endsley, 2017). Yet a survey (Dikmen \& Burns, 2016) showed that Tesla "Autopilot" users recognized the importance of understanding the limitations of automation and following safety protocols, though how they developed this recognition (e.g., from explicit instructions versus trial-and-error) was not apparent in the study's results. Because of the potential implications for safety, a pressing research need is to understand the relationship between preadoption misperceptions of automation in vehicles and postadoption behaviors over the course of developing skilled behaviors and accurate schemas with vehicle automation.

In cases for which the expectations implied by a term are misaligned with functional capabilities of automation, new or different names for the systems may be appropriate. Some manufacturers already have begun to recognize and correct potential problems with the terms used to describe automation. Mercedes-Benz has ceased to call its automated functions by the "Drive Pilot" term (examined in this study) in an effort to avoid creating inappropriate expectations. The functionality implied by the term "pilot" was of concern, because the actual capabilities of the system are driver assistance functions (McCowen, 2017). Interestingly, the research reported by Wänke et al. (2007) suggested that associations evoked by an old product name may persist even after a name change.

This experiment used online samples which cannot be assumed to represent the general population of drivers. The current study nearly exclusively sampled drivers who did not own vehicles with advanced automated functions; a sample of 


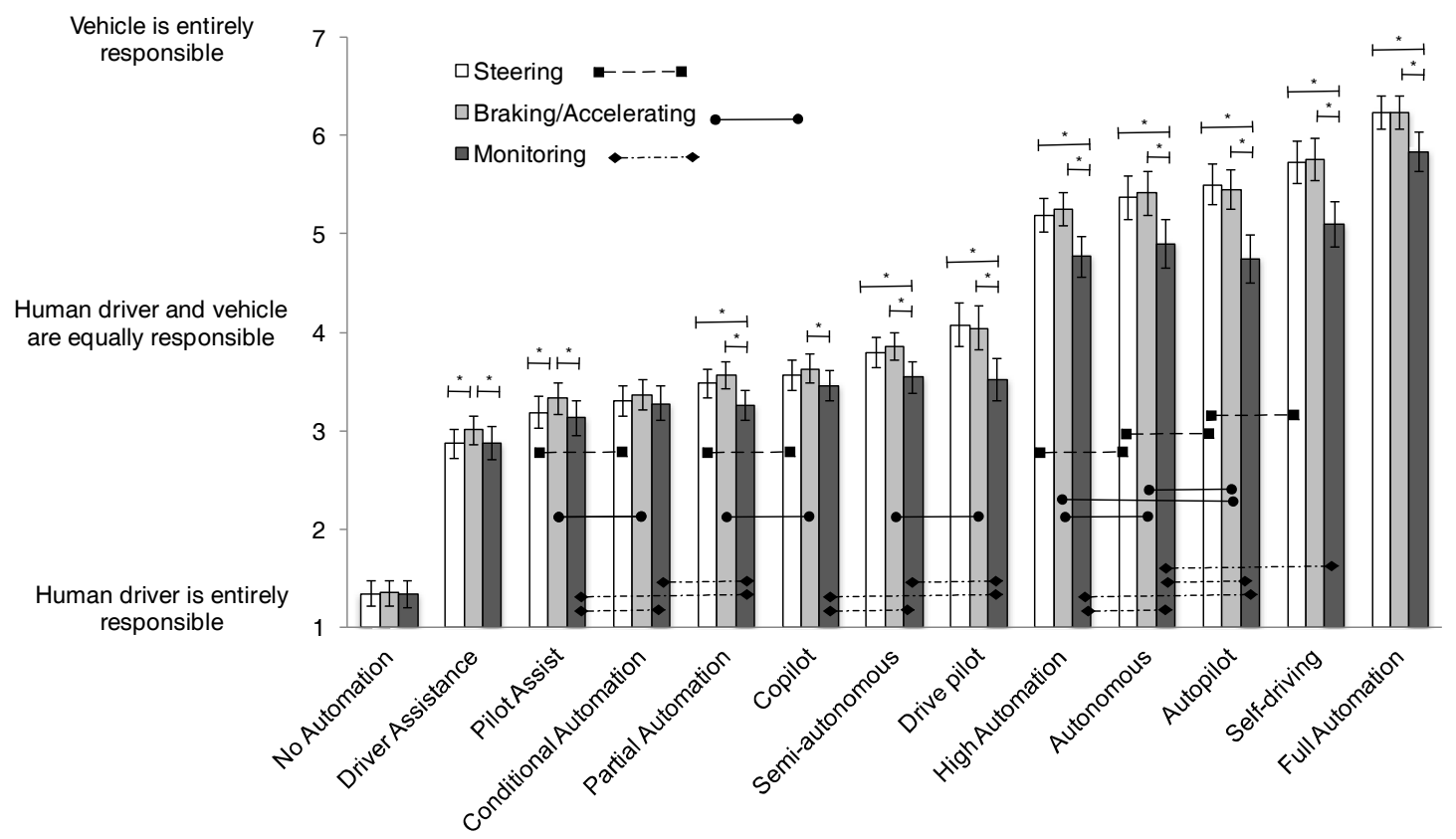

Figure 3. Simple effects analyses to examine ratings of automation terms for each DDT. Bar heights show mean ratings. For the analysis holding DDT constant and comparing across terms, differences between means are significant following Bonferroni adjustments except for bars connected by lines (dashed lines for steering, solid lines for braking/accelerating, and dotted lines for monitoring). For the analysis holding term constant and comparing DDTs, asterisks above bars indicate significant differences following Bonferroni corrections. Error bars represent $95 \%$ confidence intervals.

drivers who own or have interacted with advanced vehicle automation might produce different results. Another limitation is that peoples' perceptions of the meaning of words can change over time. The results obtained here should not be assumed to represent static rules about the perception of terms used to describe automation, but rather they give a snapshot of peoples' current understanding of an emerging and dynamic sociotechnical system. Finally, this research was exploratory in nature; more research is needed to confirm and expand upon these findings.

The current research offered support to concerns that some terms used to describe automation in vehicles imply functionality that is greater than the actual capabilities of the systems (also see Abraham et al., 2017). More research is needed to understand the perceptions of terms used to described automation, how those perceptions evolve over time, and whether a priori perceptions will affect behavior in vehicles with automation. My findings suggested, however, that the terms used to name and label automated functions in vehicles may invoke misperceptions about the technical capabilities of the vehicles.

\section{REFERENCES}

Abraham, H., Seppelt, B., Mehler, B., \& Reimer, B. (2017, September). What's in a name: Vehicle technology branding \& consumer expectations for automation. In Proceedings of the 9th International Conference on Automotive User Interfaces and Interactive Vehicular Applications (pp. 226-234).

Cole, A. (2017, February 21). What are the different levels of self-driving cars? Washington Post. Retrieved from

https://www.washingtonpost.com/cars/what-are-the-different-levels-of-selfdriving-cars/2017/02/21/444a2a80-f877-11e6-aa1e-

5f735ee31334_story.html

Dikmen, M., \& Burns, C. M. (2016, October). Autonomous driving in the real world: Experiences with tesla autopilot and summon. In Proceedings of the 8th International Conference on Automotive User Interfaces and Interactive Vehicular Applications (pp. 225-228).

Endsley, M. R. (2017). Autonomous driving systems: A preliminary naturalistic study of the Tesla Model S. Journal of Cognitive Engineering and Decision Making, 11(3), 225-238.

Hoff, K. A., \& Bashir, M. (2015). Trust in automation: Integrating empirical evidence on factors that influence trust. Human Factors: The Journal of the Human Factors and Ergonomics Society, 57(3), 407-434.

Körber, M., Baseler, E., \& Bengler, K. (2018). Introduction matters: Manipulating trust in automation and reliance in automated driving. Applied Ergonomics, 66, 18-31.

Lambert, F. (2017, May 29). People are still dangerously referring to Tesla's Autopilot as a "self-driving" system. Retrieved September 11, 2017, from https://electrek.co/2017/05/29/tesla-autopilot-dangerously-self-driving/

McCowen, D. (2017, April 19). Mercedes drops. Retrieved August 25, 2017, from http://www.drive.com.au/motor-news/mercedes-drops-pilot-name-fordriving-assistance-systems-20170413-gvkfvk.html

Nees, M. A. (2016). Acceptance of Self-driving Cars: An Examination of Idealized versus Realistic Portrayals with a Self- driving Car Acceptance Scale. Proceedings of the Human Factors and Ergonomics Society Annual Meeting, 60(1), 1449-1453.

Pak, R. (2016, October 20). Tesla is wrong to use "autopilot" term. Retrieved August 27, 2017, from http://humanfactorsblog.org/2016/10/20/tesla-iswrong-to-use-autopilot-term/

Parasuraman, R., \& Manzey, D. H. (2010). Complacency and bias in human use of automation: An attentional integration. Human Factors, 52(3), 381410.

Parasuraman, R., \& Riley, V. (1997). Humans and automation: Use, misuse, disuse, abuse. Human Factors, 39(2), 230-253.

SAE International. (2016). Taxonomy and definitions for terms related to driving automation systems for on-road motor vehicles (No. J3016) (pp. 130).

Schmitt, B. (2016, October 14). German government asks Tesla to rename Autopilot, and drivers to read their owner's manual. Retrieved August 31, 2017, from https://www.forbes.com/sites/bertelschmitt/2016/10/14/germangovernment-asks-tesla-to-rename-autopilot-and-drivers-to-read-theirowners-manual/

Simpson, J. M. (2016, November 23). Enact regulation covering advertising autonomous vehicles now. Retrieved from https://consumermediallc.files.wordpress.com/2016/11/ltrdmv112316.pdf

Wänke, M., Herrmann, A., \& Schaffner, D. (2007). Brand name influence on brand perception. Psychology and Marketing, 24(1), 1-24. 